\title{
CONCEPTOS DE SUSTANCIAS Y CONCEPTOS DE PROPIEDADES EN ANIMALES NO HUMANOS
}

\author{
LAURA DANÓN \\ Fac. de Filosofía y Humanidades y Fac. de Psicología \\ Universidad Nacional de Córdoba (Argentina) \\ Consejo de Investigaciones Científicas y Técnicas \\ ldanon@gmail.com
}

RESUMEN: El presente trabajo tiene dos objetivos centrales. Primero caracterizaré una variante de pragmatismo conceptual según la cual algunos conceptos deben entenderse como habilidades para identificar sustancias e identificar propiedades del entorno, y mostraré que quien cuenta con esas dos habilidades satisface, en grados diversos en cada caso, distintos requisitos centrales para la posesión de conceptos. Posteriormente defenderé la viabilidad de extender este enfoque a los animales no humanos, apelando a evidencia empírica que indica que distintas especies son capaces de identificar sustancias de su entorno y que sugiere la presencia de habilidades para identificar propiedades en algunos primates y pájaros.

PALABRAS CLAVE: conceptos animales, identificación, Millikan, pragmatismo conceptual, habilidades conceptuales

SUMMARY: This paper has two central aims. Firstly, I will present a variety of conceptual pragmatism according to which some concepts are abilities to identify substances and properties, showing that the creature that has these abilities satisfies, in different degrees, a group of important requirements for concept possession. Secondly, I will defend the viability of extending this approach to non human animals, by examining empirical evidence which shows: i) that different animals can identify substances in their surroundings, and ii) that some birds and primates can also identify certain functional properties.

KEY WORDS: animal concepts, identification, Millikan, conceptual abilities, conceptual pragmatism

"Concepto" es un término multívoco que cambia de significados - a menudo drásticamente - en el conjunto de disciplinas vecinas constituido por la filosofía de la mente y las ciencias cognitivas. En este trabajo, partiré de un modo de entender los conceptos que se ha denominado enfoque pragmatista (Fodor 1995). ${ }^{1}$ Para este

\footnotetext{
${ }^{1}$ Es importante indicar, brevemente, las connotaciones específicas del término "pragmatismo" en los debates sobre conceptos. Fodor fue quien introdujo, en ese contexto, la distinción entre "enfoques pragmatistas de los conceptos" y "enfoques cartesianos de los conceptos". Para el cartesiano, un concepto es un ítem mental interno cuya función principal es representar el entorno, mientras que su capacidad para guiar nuestras acciones en él es subsidiaria y secundaria. Para el pragmatista, por contraposición, poseer un concepto consiste primariamente en poseer ciertas habilidades conductuales y/o cognitivas distintivas. Esto no significa, sin embargo,
} 
enfoque, poseer un concepto consiste, fundamentalmente, en poder hacer - antes que en poder pensar - ciertas cosas, o en poseer ciertas habilidades conductuales y cognitivas distintivas. En específico, me adheriré a una variante de pragmatismo conceptual inspirada en los trabajos de Ruth Millikan, con la finalidad de aplicarla al problema de la atribución de conceptos a animales no humanos. Para ello me propongo: i) caracterizar dicha concepción de los conceptos como habilidades identificatorias; ii) mostrar que, por satisfacer un cúmulo de requisitos pertinentes, las habilidades identificatorias merecen el rótulo de conceptuales, y iii) ilustrar, mediante ejemplos empíricos, la viabilidad de extender esa caracterización para incluir a algunos animales no humanos.

Los pasos para alcanzar los propósitos mencionados son varios. En primer lugar, analizaré de manera breve la noción de habilidad y examinaré una serie de requisitos que ha de satisfacer cualquier habilidad que merezca el rótulo de conceptual. A continuación, presentaré la variante de pragmatismo conceptual que defiende: una concepción en la cual algunos conceptos han de entenderse como habilidades para identificar sustancias y propiedades del entorno. En el marco de esta propuesta, distinguiré la identificación de sustancias de la identificación de propiedades, caracterizando la primera como una tarea cognitiva más básica, e independiente de la segunda. Luego mostraré que, pese a su carácter primitivo, la identificación de sustancias satisface en un grado relevante los distintos requisitos para el dominio de conceptos y que, a su vez, la habilidad para identificar propiedades potencia y enriquece las capacidades cognitivas de una criatura de un modo que le permite satisfacer tales requisitos de manera aún más plena. Por último, vincularé estas distinciones teóricas con evidencia empírica que indica que diferentes especies no humanas son capaces de identificar sustancias de su entorno, y que

que el pragmatista conceptual deba evitar todo uso de la noción de representación mental. Millikan (2000) ha defendido, por ejemplo, la posibilidad de integrar habilidades y representaciones del siguiente modo: podemos pensar que cada concepto es idéntico - al menos en el nivel de los ejemplares - a alguna representación mental siempre que entendamos que: a) lo que determina el contenido de tal representación es la función que debe desempeñar; y b) para el cumplimiento de dicha función es preciso que el sistema cognitivo posea ciertas habilidades distintivas. Está claro que, en este punto, el pragmatismo de Millikan con respecto a los conceptos se diferencia de otros tipos de pragmatismo contemporáneo - como el de Price (2010) o el de Rorty $(1991,1997)$ - que defienden un antirrepresentacionalismo global y radical en cuanto al modo en que se vinculan el pensamiento y el lenguaje por un lado, y el mundo por otro. 
sugiere la presencia de habilidades para identificar propiedades en algunas especies de primates y pájaros.

\section{Habilidades conceptuales y requisitos para el dominio de conceptos}

Las habilidades pueden caracterizarse como capacidades, que se actualizan en circunstancias determinadas, para llevar a cabo conductas relativamente estables. Poseer la habilidad para hacer $X$ es ser capaz de realizar $X$ de modo correcto o exitoso, siempre que se cumpla algún conjunto de condiciones específicas. Pero esto no implica dar siempre, de modo rígido, las mismas respuestas conductuales, sino ser capaz de ajustarse flexiblemente a las modificaciones del medio y de las circunstancias. Además, al menos en sus casos típicos, las habilidades presentan varios rasgos que merecen especificarse. En primer lugar, las habilidades por lo general no se adquieren de golpe, sino mediante un proceso gradual que conlleva aprendizaje y admite perfeccionamiento (Ryle 1949, Millikan 2000). ${ }^{2}$ Un segundo aspecto distintivo es el carácter normativo de las habilidades. Siempre podemos fracasar en el ejercicio de una habilidad, o tener un desempeño deficiente en ella. Además, poseer una habilidad no sólo entraña actuar de manera correcta según ciertos criterios, sino ajustarse uno mismo a ellos, lo cual se manifiesta en la capacidad para monitorear el propio desempeño y reconocer los errores y corregirlos. Por último, suele añadirse que el ejercicio de una habilidad consiste en hacer algo activamente - $-\mathrm{y}$ no meramente en que nos ocurra algo - lo que supone un carácter propositivo o intencional. De allí que quien posee una habilidad ha de ser capaz tanto de desplegarla, como de abstenerse de hacerlo, de modo flexible y versátil, según sus propósitos y circunstancias (Glock 2008).

En síntesis: las habilidades son flexibles, normativas, intencionales, propositivas, graduales, aprendidas y perfectibles. Quedan fuera de esta lista, sin embargo, otros rasgos que en varias obras en la materia se han asociado con la posesión de conceptos. Es preciso, por lo tanto, añadir nuevas constricciones con el fin de recortar del conjunto de las habilidades el subgrupo de las habilidades conceptuales. Para ello presentaré en seguida algunos requisitos adicionales que

${ }^{2}$ Esto no implica que las habilidades carezcan de todo componente innato. Mi caracterización sólo pretende excluir de ese ámbito ciertos mecanismos no cognitivos como los reflejos y los mecanismos de descarga innatos (Bermúdez 2003), que producen respuestas invariantes a estímulos específicos con independencia de la historia individual de la criatura. 
reúnen buena parte de las intuiciones nucleares sobre los conceptos y que tanto el sentido común, como la literatura filosófica y científica contemporánea comparten. ${ }^{3}$

\section{a) El requisito de intencionalidad}

Los conceptos se caracterizan por su intencionalidad o acerquidad. Esto es, por versar sobre cosas distintas de ellos mismos. Esto implica: i) que poseen un referente, y ii) que representan tal referente de cierto modo o en cierto aspecto.El referente de un concepto es el conjunto de elementos a los que éste se aplica. Pero, además de hacer referencia a ciertos elementos, un concepto representa de un modo específico a su referente, como poseedor de un conjunto de rasgos característicos. Poseer el concepto de $X$ no se reduce a pensar acerca de los $X$; entraña, además, la capacidad para pensar en $X$ en cierto aspecto.

\section{b) El requisito de distancia}

Los filósofos suelen mencionar como rasgo distintivo del dominio conceptual la existencia de algún tipo de "distancia" o "separación" entre quien posee conceptos y aquello a lo que éstos hacen referencia (Camp 2009, Bermúdez 2003, Allen 1999). Si $S$ cuenta con el concepto $X, S$ ha de ser capaz de pensar en $X$ con independencia de sus circunstancias actuales y de los estímulos perceptuales que tengan un impacto inmediato en él. Ha de haber, pues, una distinción clara entre la representación y lo representado, que le permita representarse a $X$ aun cuando $X$ no se encuentre presente en el entorno cercano.

Ahora bien, es posible distinguir varios modos en que una criatura puede independizarse de los estímulos del entorno. Entre los menos exigentes se encuentran: la formación de expectativas acerca del futuro, el empleo de información adquirida en el pasado que no resulta perceptible actualmente y la representación de objetos, sucesos y situaciones distantes en el espacio. Pero existen maneras más radicales de distanciarse cognitivamente del entorno, como la capacidad para representar objetos, situaciones, etc., no vinculadas directamente con las necesidades propias actuales o con los objetivos primarios, y la capacidad para pensar en algo con lo que no se ha tenido contacto

\footnotetext{
${ }^{3}$ No pretendo, sin embargo, que el conjunto de constricciones que ofrezco sea exhaustivo, ni que dé cuenta de todos los rasgos pertinentes que se otorgan a los conceptos.
} 
perceptual alguno. Una criatura puede satisfacer entonces el requisito de distancia en diversos grados, dependiendo de los modos en que logra independizarse, mediante el pensamiento, de su entorno inmediato. ${ }^{4}$

\section{c) El requisito de generalidad}

Un supuesto muy extendido entre los filósofos es que nuestros conceptos constituyen las unidades mínimas del pensamiento, que se combinan entre sí de manera sistemática para formar estructuras más complejas. Empleando la jerga de las habilidades, podemos decir que la posesión de pensamientos complejos depende de la capacidad para ejercer habilidades representacionales más simples, recombinándolas entre sí. Así, si alguien es capaz de pensar que el niño es feliz, es porque puede pensar de manera independiente en el niño y en la propiedad ser feliz (Camp 2009). Si, efectivamente, los conceptos son unidades recombinables que podemos "manipular" de modo independiente, ha de ser posible para su propietario combinarlos entre sí de manera general formando con ellos todo tipo de estructuras representacionales complejas. Esto es, en esencia, lo que exige el requisito de generalidad (RG), que Evans formuló originariamente en los siguientes términos:

RG: $\mathrm{Si}$ es posible atribuir a un sujeto el pensamiento de que $a$ es $F$, entonces debe poseer los recursos conceptuales para pensar que $a$

${ }^{4}$ La idea de que el requisito de distancia, como todos los otros, puede satisfacerse en variantes más o menos débiles es consonante con mi propósito de desarrollar una concepción gradualista de los conceptos. Es evidente que no todos los filósofos estarían de acuerdo con esta estrategia. En particular, pienso que algunos argumentos de Davidson y McDowell en contra del pensamiento animal descansan en la idea de que, para poseer conceptos y pensamientos, una criatura debe poder distanciarse de su entorno de un modo especialmente radical: volviéndose reflexivamente sobre sus propias creencias para evaluar sus "credenciales epistémicas" (McDowell 1994) o para considerar si son verdaderas o falsas (Davidson 1982). Sin embargo, es posible hallar críticas fuertes a estos argumentos (Camp 2009, Lovibond 2006, Child 1994). Por mi parte, en "Discriminar, identificar, conceptualizar" (2008) defendí que la posición de Davidson lo obliga a adherirse a una dicotomía estricta entre los humanos que poseen conceptos y los otros animales que sólo discriminan rígidamente su entorno, dicotomía que no logra acomodar adecuadamente mucha de la evidencia empírica disponible. De manera similar, McDowell se ve comprometido a distinguir tajantemente entre los humanos que pueden volverse sobre sus creencias para corregirlas activamente y el resto de los animales, que carecen absolutamente de tales capacidades. En otro texto (Danón 2011), sin embargo, he defendido que podemos distinguir una capacidad no reflexiva para detectar errores en nuestras propias creencias y corregirlos, la cual resulta, al menos en principio, asequible a animales no humanos, carentes de lenguaje. 
es $G$, para cada propiedad de ser $G$ de la que tenga una concepción. (Evans 1982, p. 104)

Se suele señalar que una virtud del requisito de generalidad es que permite dar cuenta de la productividad del pensamiento humano. Un rasgo sorprendente de la cognición humana es la capacidad para elaborar y comprender innumerables pensamientos novedosos. Ahora bien, resulta difícil explicar cómo criaturas con recursos cognitivos limitados, que sólo pueden almacenar una cantidad finita de información, poseen una capacidad ilimitada para formar nuevos contenidos representacionales. RG da una solución a esta dificultad, pues permite explicar la productividad del pensamiento como resultado de nuestra habilidad para recombinar de modos innovadores y variados un repertorio acotado de conceptos.

\section{d) El requisito de potencialidad inferencial}

Se suele aceptar que quien posee conceptos ha de poder emplearlos en distintos tipos de inferencias que le permitan ampliar su conocimiento del entorno y ajustar mejor su conducta a él. Quien domina el concepto perro y sabe que Fido es un perro, puede inferir que Fido es un animal, etc. Éste es el núcleo de lo que denominaré el requisito de potencialidad inferencial, el cual admite versiones en mayor o en menor grado demandantes, dependiendo de: i) qué noción de inferencia se adopte, y ii) qué grado de generalidad se exija a las capacidades inferenciales del usuario de conceptos.

En relación con (i), a menudo se defiende que las habilidades inferenciales dependen del dominio de un lenguaje natural y se hallan fuera de la esfera de competencia de los animales no humanos. Tal tesis suele apoyarse en una concepción de la inferencia como el paso de un conjunto de premisas a una conclusión, cada una de las cuales requiere necesariamente articulación lingüística (Vigo y Allen 2009). Sin embargo, otros autores defienden concepciones más laxas de inferencia, independientes del dominio de un lenguaje natural (Watanabe y Huber 2006). Entre ellos, Call (2006a, 2006b) sostiene que los procesos inferenciales se caracterizan por la asociación de dos eventos: uno perceptible y otro no accesible a la percepción inmediata. Así entendidas, las inferencias no suponen, necesariamente, el paso de enunciados que operan como premisas a un enunciado que cumple el papel de conclusión, pero sí requieren que la criatura realice una actividad cognitiva que exceda la mera asociación de sucesos externos 
y la identificación de regularidades entre ellos. Tal actividad consiste en anticipar un rasgo no observable a partir de las propiedades observables de los fenómenos con los que la criatura se encuentra.

En cuanto a (ii) — cuán amplias han de ser las capacidades inferenciales de una criatura para atribuirle conceptos-, suele defenderse que el dominio conceptual requiere la posibilidad de realizar inferencias plenamente generalizables a los más diversos contextos. En oposición a tales ideas, otros autores nos advierten sobre el riesgo de "sobreintelectualizar" lo mental al adherirse a una concepción de la racionalidad como un fenómeno de "todo o nada" (Hurley 2006, Watanabe y Huber 2006). Ellos defienden, en cambio, una concepción gradualista de la racionalidad, según la cual muchas capacidades inferenciales de distintas especies no son más que competencias de dominio específico, que se aplican en contextos acotados.

A mi entender, los principios examinados reúnen buena parte de los rasgos que se atribuyen a los conceptos en la discusión contemporánea. El requisito de intencionalidad garantiza su carácter referencial y aspectual. El requisito de distancia capta nuestra intuición de que el pensamiento conceptual ha de poder ser pensamiento sobre lo ausente, lo no real y lo distante en el tiempo y el espacio. El requisito de generalidad apresa la idea de que nuestros conceptos son herramientas cognitivas que se articulan entre sí de modos diversos, lo que permite construir pensamientos complejos y variados. Por último, el requisito de potencialidad inferencial subraya que la posesión de conceptos se acompaña de la capacidad para realizar con ellos inferencias que guíen nuestra conducta. De allí que, si una criatura posee un conjunto de habilidades cognitivas que satisfacen esos requisitos, parece apropiado - de acuerdo con un uso relativamente estándar - caracterizarlas como habilidades conceptuales.

Ahora bien, todavía hace falta hallar algún tipo de habilidad cognitiva que satisfaga estas constricciones. Con ese objetivo en mente, dedicaré la próxima sección a caracterizar dos tipos de habilidades específicas - la identificación de sustancias y la de propiedades- y a mostrar cómo logran satisfacer los requisitos pertinentes.

\section{La identificación de sustancias y de propiedades como habilidades conceptuales}

Entre los aportes teóricos más destacados de Ruth Millikan (1998a, $1998 b, 2000)$ se encuentra la elaboración de una teoría novedosa de los conceptos, que difiere en puntos centrales de las teorías filosóficas y psicológicas tradicionales. Por lo general, éstas identifican el 
empleo de conceptos con la clasificación, que consiste en subsumir un particular bajo una clase que se define por un conjunto de propiedades. Tal proceso supone la capacidad para pensar de manera independiente en las propiedades que definen la clase y para atribuirlas al caso que se pretende clasificar (Millikan 2000). A diferencia de tales enfoques, Millikan otorga un papel central, aunque no exclusivo, a los conceptos de sustancia, a los que caracteriza en términos de habilidades para identificar y reidentificar sustancias de nuestro entorno, reconociéndolas como las mismas en nuestros sucesivos encuentros con ellas y en condiciones diversas. ${ }^{5}$ En diferentes ocasiones, la misma sustancia se nos manifestará desde distintas perspectivas, por medios diferentes, en tiempos y lugares variables, etc. La identificación consistirá en la capacidad para reconocer que en todos esos casos estamos ante lo mismo otra vez.

En la categoría de sustancia, Millikan agrupa individuos, clases (naturales y artificiales) y materiales de diversa índole. Pese a sus diferencias, todas estas variedades de sustancia comparten un rasgo común: mantienen al menos algunas propiedades a lo largo del tiempo. De allí que la habilidad para identificar sustancias permita acumular conocimiento teórico y práctico sobre tales rasgos estables y aplicarlo, sin que pierda validez, a encuentros futuros con ellas. Dado que, a menudo, una sustancia manifiesta algunas propiedades en ciertas circunstancias y no en otras, almacenar este tipo de conocimiento resulta especialmente útil, pues nos permite ajustar nuestro comportamiento a ciertos rasgos que, de momento, no se manifiestan perceptualmente.

Aunque los conceptos de sustancia no agotan nuestro repertorio conceptual, sí desempeñan una función más básica que otros conceptos, como los de propiedades. Identificar una sustancia no requiere necesariamente la posesión previa de conceptos de propiedades. Por el contrario, esa identificación sólo exige que la criatura sea sensible y responda de modo apropiado a los rasgos de la sustancia, aunque no pueda representarse aisladamente cada una de sus propiedades ni recombinarlas espontáneamente en su pensamiento con otras entidades del entorno ( $c f r$. Millikan 2000, p. 77). De allí que un niño pequeño pueda reconocer a su madre por su olor sin ser capaz de pensar de manera independiente en la propiedad tener el olor X. En

\footnotetext{
${ }^{5}$ Millikan alterna el uso de las expresiones "identificar" y "reidentificar", dándoles sentidos equivalentes (Millikan 2000, p. 144). Por lo general, emplearé sólo el primero de estos vocablos, para simplificar la lectura.
} 
estos casos "el pensamiento es acerca de la mamá, no del olor, pero surge en respuesta al olor" (Millikan 2000, p. 81). ${ }^{6}$

En criaturas de cognición más sofisticada, se añade a la identificación de sustancias la habilidad más tardía para identificar propiedades en lugar de limitarse a responder a ellas de modos específicos (Millikan 1984). Pero, ¿en qué consiste la identificación de una propiedad y en qué se diferencia de la identificación de sustancias? Ofreceré aquí una respuesta a tales preguntas que es en buena medida propia, aunque recupera las breves observaciones de Millikan sobre este tema.

Si establecemos una analogía entre la habilidad para identificar propiedades y la habilidad para identificar sustancias, parece que, mientras que esta última consiste en poder tratar una sustancia como la misma aun cuando ocasionalmente varíen algunas de sus propiedades, la primera consiste en poder reconocer una propiedad como la misma, aun cuando aparezca instanciada en diversas sustancias. De esta manera, quien identifica una propiedad como blanco ha de detectar que se encuentra ante el mismo color una y otra vez, sin importar si éste aparece como propiedad de un mantel, del algodón o de la nieve.

Por otra parte, como señalan Cangelosi y Parisi (1998), las propiedades también parecen contar con cierto potencial inductivo, aunque éste sea menor que el de las sustancias. Sabemos, por ejemplo, que lo que es blanco se ensucia con más frecuencia que lo de colores oscuros, se mantiene más fresco bajo el sol, se distingue fácilmente aunque la luz sea tenue, etc. De allí que la identificación de propiedades también permita acumular conocimiento y realizar algunas inferencias adecuadas.

Hay que añadir que, para Millikan (1984), una propiedad se caracteriza fundamentalmente por oponerse a otras propiedades contrarias, incompatibles con ella. Así, lo que es rojo no puede, al mismo tiempo, ser azul o verde, lo que está debajo de una cosa no puede estar sobre

${ }^{6}$ He aquí de nuevo la tesis de Millikan según la cual la posesión de conceptos de sustancias, tal como ella los entiende, es anterior a la posesión de conceptos de propiedades e independiente de ésta (Millikan 2000). Es importante notar, sin embargo, que ésta es una tesis sobre la prioridad lógica y psicológica de los conceptos de sustancias sobre los conceptos de propiedades y no una tesis metafísica sobre la prioridad de las sustancias sobre las propiedades qua elementos de un mundo independiente de la mente. En este sentido, aún queda por resolver en qué medida podría compatibilizarse la teoría de Millikan con distintas posiciones metafísicas sobre el vínculo ontológico existente entre sustancias y propiedades, y esto merece un debate en detalle. 
ella, etc. ${ }^{7}$ En la medida en que la identidad de una propiedad depende de su oposición a otras propiedades contrarias, la habilidad para identificar una propiedad exigirá que seamos capaces de identificar también las propiedades contrarias y de reconocer que la primera y las segundas nunca se presentan simultáneamente en un mismo objeto (Millikan 1984).

Habiendo caracterizado la identificación de sustancias y de propiedades, podemos preguntarnos si dichas habilidades satisfacen los requisitos pertinentes para calificarlas de conceptuales. En lo que sigue intentaré mostrar que ambas habilidades logran satisfacer los requisitos para la posesión de conceptos, aunque en diferentes grados. La criatura que identifica sustancias satisface variantes moderadas de los diversos requisitos, lo cual permite atribuirle conceptos, aunque sea en un sentido modesto. Por otra parte, la habilidad para identificar propiedades amplía y potencia las capacidades cognitivas y conductuales de una criatura, y le permite satisfacer aún con mayor plenitud las constricciones en cuestión. En consecuencia, quien identifica propiedades poseerá habilidades conceptuales más ricas y complejas, que podrá emplear de modos novedosos, inaccesibles para quien sólo identifica sustancias.

En primer lugar, parece claro que tanto la identificación de sustancias como la de propiedades poseen los rasgos que presenté como característicos de una habilidad. Una criatura puede ser más o menos competente en ambos tipos de identificación y puede cometer errores al realizar tales tareas. A su vez, esos errores pueden conducirla a realizar inferencias equivocadas y a comportarse de modos inadecuados. Hay que añadir a lo anterior que, por lo general, la criatura puede percatarse de tales tropiezos, corregirlos y perfeccionar sus habilidades identificatorias. Finalmente, la identificación de sustancias o propiedades suele ser flexible y ajustarse a nuestros intereses y propósitos. Estamos, pues, ante habilidades típicas: normativas, perfectibles, aprendidas, versátiles y propositivas.

Veamos ahora qué ocurre con los requisitos adicionales asociados con el dominio conceptual. De acuerdo con el requisito de intencionalidad, los conceptos han de poseer un referente y han de representarlo en cierto aspecto. Sin duda, las habilidades identificatorias satisfacen esta condición de referencialidad, pues versan sobre las

${ }^{7}$ En palabras de Millikan: "Una propiedad se encuentra, en sí misma, en una oposición naturalmente necesaria con el resto de una gama de propiedades o de contrarios en la cual entra, de modo que cualquier sustancia que posea esa propiedad no puede tener, de acuerdo con una necesidad natural, ninguna de las propiedades del resto de esa gama" (Millikan 1984, p. 271). 
sustancias o propiedades que la criatura tiene por función identificar. En cambio, al menos a primera vista, la teoría de Millikan parece enfrentar dificultades al momento de incorporar la aspectualidad de nuestros conceptos. Veamos por qué.

Al desarrollar su teoría de los conceptos, Millikan (2000) distingue dos modos de individuar nuestras habilidades identificatorias: por sus fines o por sus medios. En el primer caso, lo que importa es qué sustancias somos capaces de identificar. En el segundo nos interesa, además, qué medios empleamos para lograr tal fin, qué información acumulamos y qué inferencias podemos realizar. Millikan reserva el término "concepto" para el primer modo de considerar las habilidades y la palabra "concepción" para el segundo. Así, cuando decimos que una criatura posee el concepto de $X$, sólo afirmamos que es capaz de identificar los $X$, sin importar por qué medios lo logre.

Podría parecer que esta propuesta descuida la aspectualidad de los conceptos, pues lo único relevante al momento de individuarlos es su referente. Pero, aunque la identificación de una sustancia puede realizarse empleando diversos medios, en cada caso particular depende de algún medio específico, entraña cierta información y permite realizar algún conjunto de inferencias. Nunca hay conceptos "en el aire" que no se encuentren acompañados de alguna concepción. Pese a su énfasis en lo que tienen en común las criaturas que identifican un mismo referente, la teoría de Millikan no niega que aquello que identificamos se presente siempre con un aspecto determinado y dispone de los recursos teóricos para dar cuenta de este rasgo de los conceptos.

Examinemos ahora una segunda interrogante: ¿satisfacen el requisito de distancia las criaturas que identifican sustancias? Hay al menos dos sentidos en los que quienes poseen tal habilidad logran distanciarse de su entorno inmediato. En primer lugar, como vimos, identificar una sustancia conlleva la capacidad para obtener información sobre rasgos suyos que se manifiestan ocasionalmente y para aplicar ese conocimiento a situaciones en las que éstos no resultan perceptibles. Luego, quien identifica sustancias no se encuentra confinado a representarse los objetos exactamente como se presentan ante su percepción, en su aquí y ahora inmediato, sino que puede disponer de información adquirida en el pasado y emplearla para anticipar la conducta futura de las entidades con las que interactúa.

Por otra parte, según Millikan, mientras que una criatura que sólo posee habilidades perceptuales puede "rastrear" un objeto mediante el olfato, la vista, el oído, etc., percibiéndolo como el mismo en condiciones distintas, la criatura que cuenta con habilidades conceptuales 
puede reidentificar una sustancia aun cuando el rastreo perceptual se vea interrumpido por largo tiempo. ${ }^{8}$ En consecuencia, logra satisfacer el requisito de distancia en un segundo sentido: aunque no perciba por largos periodos un objeto, puede continuar "rastreándolo conceptualmente", entendiendo que se trata del mismo y reidentificándolo posteriormente ( $c f r$. Millikan 2000, p. 78).

Ahora bien, hay al menos un punto respecto del cual quienes sólo poseen conceptos de sustancias no satisfacen el requisito de distancia. $\mathrm{Al}$ parecer, todo el conocimiento que tales criaturas pueden acumular sobre una sustancia han de adquirirlo mediante la interacción perceptual y la manipulación directa. Por lo tanto, son incapaces de pensar en objetos ficticios o inexistentes, entidades del pasado, etcétera.

Por su parte, la habilidad para identificar propiedades aumenta la distancia e independencia de los pensamientos de una criatura respecto de los estímulos de su entorno, pues permite acumular información y realizar inferencias acerca de una propiedad con independencia de las sustancias particulares en las que se instancia. Además, cabe conjeturar que esta habilidad posibilita el surgimiento de una capacidad cognitiva ulterior: pensar en objetos nuevos con los que no se ha interactuado previamente. La idea es la siguiente: al poder reconocer, adquirir información y realizar inferencias sobre propiedades como blanco, agudo, enojado, etc., con independencia de las sustancias en las que se instancien, se abre la posibilidad de manipular mentalmente esas propiedades, combinándolas de modos novedosos, y de imaginar así objetos diferentes de aquellos que se presentan en nuestro entorno.

Veamos, a continuación, qué ocurre con el requisito de generalidad. ¿Pueden satisfacerlo las criaturas que sólo identifican sustancias? La respuesta a esta pregunta es compleja y requiere el análisis previo de distintas lecturas posibles del requisito. Según la formulación canónica de Evans, RG presupone que nuestras habilidades conceptuales se combinan formando estructuras del tipo $a$ es $G$ en las cuales se aplica un predicado $G$ a un sujeto particular $a$. Si esto es así, resulta claro que los pensamientos de la criatura que sólo identifica sustancias, pero carece de conceptos de propiedades, no poseen el tipo de estructura proposicional que se requiere para satisfacer RG. Por el contrario, parece más adecuado entender los conceptos

${ }^{8}$ De todos modos, es importante recordar que, según Millikan (2000), la distinción entre habilidades perceptuales y conceptuales sólo es de grado, y que las primeras constituyen la base a partir de la cual logran erigirse, paulatinamente, las segundas. 
de sustancia que posee como habilidades para detectar patrones de manera holista, respondiendo a ciertos rasgos de los objetos, pero sin poder pensarlos como propiedades separadas de la totalidad en la que aparecen.

Ésta parece una buena razón para rechazar la posibilidad de que las criaturas que sólo cuentan con conceptos de sustancias satisfagan RG. Sin embargo, creo que si nos focalizamos en lo que la criatura que posee conceptos de sustancias puede hacer con ellos —antes que en su incapacidad para formar un tipo específico de contenidos: los proposicionales - podemos matizar tal conclusión. Como se señaló antes, quien posee el concepto de una sustancia $X$ puede identificar $X$ por diversos medios, respondiendo a distintos rasgos de la misma. Así, un perro puede identificar un hueso y tratarlo como un objeto con el cual puede jugar, como alimento, etc. Luego, resulta natural atribuirle contenidos mentales como el hueso está enterrado, el hueso es un alimento, etc., en función de los rasgos del hueso a los que esté respondiendo.

Por otra parte, si una criatura es capaz de discriminar ciertas propiedades de la sustancia $X$ cabe esperar, al menos en principio, que cuente con una capacidad análoga para aprender a detectar esos mismos rasgos cuando se presenten en alguna otra sustancia. Así, si tenemos un perro que sabe jugar con huesos y lo dejamos interactuar con una rama, es probable que pronto aprenda a tratarla como un juguete. En tales casos, parecerá sensato atribuirle contenidos como el hueso es un juguete y la rama es un juguete.

Sin duda, al atribuir tales contenidos mentales los intérpretes nos vemos obligados a emplear nuestro lenguaje natural, componiendo estructuras con el formato sujeto-predicado en las cuales ciertos conceptos de propiedades se aplican a un sujeto. Pero no es preciso que esta estructura proposicional refleje la estructura de los contenidos que de hecho poseen los animales sin lenguaje. Alternativamente, es posible pensar que estamos usando una herramienta lingüística de articulaciones finas y complejas para rastrear las modificaciones que se producen en los contenidos no lingüísticos, más toscos y globales, de esas criaturas. ${ }^{9}$ Tales modificaciones no podrán explicarse en

${ }^{9}$ Es importante subrayar que no pretendo adoptar aquí una concepción interpretativista/instrumentalista con respecto a los contenidos mentales que atribuimos a quien sólo identifica sustancias, pues no pienso que tales contenidos sean meras "ficciones útiles" para explicar su conducta. Antes bien, mi idea es que las criaturas que sólo identifican sustancias cuentan, de hecho, con contenidos mentales que versan acerca de esas sustancias y sus rasgos, aunque no estén compuestos exclusivamente por la combinación de habilidades conceptuales, ni posean la estructura proposicional 
términos de su capacidad para combinar conceptos de particulares con conceptos de propiedades, pero sí quizás en términos de: i) la habilidad general para identificar ciertas sustancias; ii) un conjunto de competencias para registrar, discriminar y responder conductualmente a ciertos rasgos en distintas sustancias. ${ }^{10}$

Según estas consideraciones, en una lectura estándar, el RG no puede aplicarse a una criatura que sólo posea conceptos de sustancias. Pero sí es posible pensar que tal criatura satisface una variante más laxa de RG, que no impone restricciones con respecto a la estructura (proposicional o no) de sus pensamientos, ni exige que éstos sean el resultado de la combinación de dos o más habilidades conceptuales; lo único que exige es que la criatura sea capaz de formar pensamientos variados acerca de las sustancias que es capaz de identificar y de las propiedades que logra discriminar en dichas sustancias. Además, no es necesario suponer que la criatura posee conceptos de las propiedades que discrimina. ${ }^{11}$

Es necesario añadir, sin embargo, otra constricción con respecto al modo en que podría satisfacer el RG quien sólo identifica sustancias. Camp (2009) distingue dos variantes distintas del RG. Según la más exigente, quien posee conceptos ha de poder recombinarlos espontáneamente, de todas las formas posibles, con independencia de los estímulos del entorno que repercutan en él. La segunda variante, que Camp bautiza como causal-contrafáctica, sólo exige que la criatura pueda combinar los conceptos de su repertorio si se presentan en el entorno los estímulos pertinentes. Ahora bien, he sugerido que la criatura que sólo identifica sustancias puede formar nuevos pensamientos en los que se combinen de distintos modos sus conceptos

con las que nuestras atribuciones lingüísticas los revisten. Agradezco a un árbitro anónimo de Crítica el haberme hecho reparar en este punto.

${ }^{10}$ No debemos confundir esta capacidad para discriminar propiedades con la habilidad para identificarlas. El animal que discrimina una propiedad responde de modo específico e invariante cuando ésta se presenta en cierta sustancia. En cambio, quien la identifica es capaz de acumular distinta información sobre la propiedad, realizar inferencias y combinarla con otras propiedades, separándola de las sustancias particulares en las que la haya detectado en el pasado.

${ }^{11}$ Cabe preguntarse si, al debilitarlo de este modo, el RG no deja de cumplir con la tarea originaria para la cual Evans lo propuso: trazar una distinción nítida entre el contenido conceptual y el no conceptual. Sin embargo, mi interés en este trabajo no es emplear el RG para distinguir entre tipos de contenidos, sino como un requisito - entre otros - para dirimir qué criaturas poseen (algunos) conceptos (Camp 2009 y Carruthers 2009 emplean el RG de manera similar). Éste es, según pienso, un propósito diferente e independiente del de precisar si los contenidos mentales de esta criatura están compuestos enteramente por constituyentes conceptuales - como sostienen los defensores del contenido conceptual-, o no. 
(de sustancia) con su capacidad para registrar y responder a ciertas propiedades en esas sustancias. No obstante, tal capacidad para formar nuevos pensamientos dependerá estrictamente de los estímulos del ambiente. Sólo podrá, por ejemplo, tratar un nuevo objeto como juguete si al manipularlo aprende que se presta a tal uso. Debido a su imposibilidad para pensar en las propiedades de manera independiente, esa criatura será incapaz de combinar, espontáneamente de modos novedosos, propiedades y sustancias que no se hayan presentado ante su percepción. Luego, sólo logrará satisfacer el RG de modo causalcontrafáctico, cuando el entorno le provea los estímulos adecuados para ello.

Esos límites desaparecen para la criatura que identifica propiedades, pues ésta sí puede manipular espontáneamente los conceptos de propiedades y de sustancias que posee, inventando combinaciones novedosas con las que nunca se ha tropezado en su entorno. De este modo, quien identifica propiedades logra satisfacer el requisito de generalidad en un sentido más vigoroso. ${ }^{12}$

Detengámonos, por último, en el requisito de potencialidad inferencial. Como se ha señalado, la habilidad para identificar una sustancia entraña la capacidad para efectuar inferencias sobre su comportamiento futuro, apoyándose en información sobre ella adquirida previamente. Pero también esta potencialidad inferencial aumenta de modo notable para quien identifica propiedades, pues este individuo puede acumular información general y abstracta sobre ciertas propiedades, y aplicarla a distintas sustancias, aun cuando no haya tenido una historia de interacciones pasadas con ellas. Pensemos, por ejemplo, en una criatura capaz de detectar la propiedad estar enojado que sabe, además, que ésta suele venir acompañada de con-

${ }^{12}$ Esto no implica que toda criatura que posee conceptos de propiedades deba ser capaz de recombinar de modo pleno todos sus conceptos de particulares con sus conceptos de propiedades. Otras constricciones presentes en distintas especies - como la incapacidad para representar hechos carentes de utilidad práctica, o el que ciertas capacidades cognitivas sean de dominio específico- pueden introducir límites adicionales a sus posibilidades de recombinación conceptual. Éste no es un problema si, como he hecho aquí, admitimos variantes débiles del RG que no exijan la recombinabilidad plena. Una estrategia de este tipo deja abierta la posibilidad de que algunas habilidades identificatorias sean modulares y, no obstante, cuenten como conceptuales. Para ello basta que sea posible cierta recombinación intramodular y/o intermodular de las habilidades conceptuales, que éstas permitan identificar un referente en cierto aspecto y con alguna independencia del estímulo, y que permitan llevar a cabo algún conjunto acotado de inferencias. Puede hallarse una exposición más detallada sobre variantes del RG sometidas a constricciones de modularidad en Duhau 2009, pp. 57-61. 
ductas agresivas. Si se encuentra ante un animal nunca visto antes y detecta que está enojado, puede inferir que se comportará agresivamente. Sus capacidades inferenciales se vuelven, de esta manera, más amplias y generales, y se independizan de la necesidad de haber tenido experiencias pasadas con cada sustancia sobre la cual realiza inferencias.

En síntesis, partiendo de los desarrollos de Millikan he distinguido la identificación de sustancias de la identificación de propiedades y he defendido que ambas satisfacen un conjunto de requisitos que permiten calificarlas de habilidades conceptuales. Señalé también que existen diferencias relevantes en el grado y el modo en que la posesión de estas habilidades permite satisfacer los requisitos de distancia, generalidad y potencialidad inferencial. Las criaturas que únicamente identifican sustancias sólo satisfacen estos principios de manera acotada y en sus variantes débiles. Aquellas que, además, identifican propiedades pueden satisfacerlos de una forma más amplia y plena. La identificación de sustancias constituye así un primer nivel, limitado y básico, de dominio conceptual. La identificación de propiedades, en cambio, supone la posesión de un espectro más rico y vigoroso de habilidades conceptuales.

Con base en esta distinción entre conceptos de sustancias y de propiedades cabe preguntarse por su aplicación al estudio de la cognición de animales no humanos. ¿Hay otras especies que posean conceptos como los aquí caracterizados? En caso de que así fuera, ¿tienen sólo conceptos de sustancias o cuentan, además, con conceptos de propiedades? La siguiente sección se dedica a ofrecer una primera aproximación a tales problemas, a partir del análisis de alguna evidencia empírica sobre la conducta animal.

\section{Animales que identifican sustancias}

Aunque no hay aplicaciones explícitas de los planteamientos teóricos de Millikan a los estudios empíricos con animales no humanos, deseo presentar aquí alguna evidencia que, a mi parecer, indica la existencia de capacidades para identificar sustancias significativas para la propia supervivencia en distintas especies. Según se desprende de las secciones previas, tal habilidad para tratar algo como lo mismo se manifiesta con claridad en los casos en que un animal:

i) no responde ante un único tipo de estímulos indicadores de la sustancia, sino que puede reconocerla por diferentes medios y en circunstancias variables; 
ii) no responde rígidamente a la sustancia, sino que modifica su conducta en función de sus interacciones previas y de la información que haya obtenido sobre ella en el pasado.

Apoyándome en estos criterios, analizaré algunos ejemplos empíricos que se ajustan a la propuesta teórica de Millikan de un modo especialmente sugerente.

\subsection{Identificación de individuos en primates no humanos}

La etología cognitiva proporciona amplia y variada evidencia de que, en sus entornos naturales, distintas especies de primates reconocen a otros individuos, almacenan información sobre ellos y la emplean para inferir su comportamiento futuro. He aquí algunos comportamientos indicadores de tales competencias. ${ }^{13}$

\section{a) Conductas diferenciales hacia los miembros de la propia familia materna}

De acuerdo con diversos primatólogos, numerosas especies de primates identifican a sus parientes por vía materna, y los diferencian de los otros miembros del grupo (Kapsalis 2004). Destacan aquí los estudios de Cheney y Seyfarth $(1990,2007)$ que muestran cómo los monos vervet y los babuinos son capaces de reconocer a sus parientes maternos y mantener con ellos un vínculo social estrecho, tratarlos de manera diferenciada y, en respuesta, esperar de ellos ciertas disposiciones conductuales típicas, por ejemplo, una mayor tendencia a aliarse en peleas contra terceros y a esperar su ayuda cuando son atacados, una marcada tendencia a espulgarse mutuamente y una mayor cantidad de interacciones cooperativas.

\section{b) Conductas de reciprocidad entre miembros de un grupo}

Las observaciones etológicas y los experimentos realizados en entornos naturales coinciden en señalar que numerosos primates dan respuestas cooperativas - como espulgarse, compartir alimentos o aliarse en una pelea contra otros monos - a los individuos que han cooperado con ellos en el pasado. Así, en distintas especies de primates que viven en grupos - como los vervet, los monos capuchinos,

${ }^{13}$ Hay que aclarar, sin embargo, que en ninguno de los estudios citados los investigadores aplicaron explícitamente categorías como concepto de sustancia o habilidades identificatorias, ni para diseñar sus experimentos, ni para interpretar sus datos. 
los chimpancés, los mandriles y los macacos japoneses- se ha encontrado una correlación positiva entre el espulgamiento y el apoyo posterior mediante alianzas (Ventura et al. 2006, Cheney y Seyfarth 1990, Cords 1997). Además, en distintos primates se ha observado, a modo de retorno recíproco, la tendencia a espulgar más a los miembros de su grupo que los espulgaron a ellos en el pasado (Ventura et al. 2006).

\section{c) Conductas de reconciliación}

En criaturas muy sociales, como los primates no humanos, los conflictos son frecuentes y pueden tener consecuencias disruptivas para la estabilidad grupal o para la red de relaciones de cada individuo. Probablemente como modo de reparar o morigerar tales consecuencias, se han observado en distintos primates conductas de "reconciliación" después de un encuentro agresivo. Expongo a continuación algunos ejemplos.

En los babuinos es frecuente que, cuando una hembra de alto rango ha atacado a una de rango inferior, la primera se dirija luego a la segunda gruñendo con suavidad. Los investigadores piensan que esos gruñidos operan como una señal que permite a la víctima concluir que el conflicto ha finalizado, y esto favorece las interacciones pacíficas futuras entre las antiguas rivales (Cheney y Seyfarth 2007).

También los chimpancés buscan activamente contacto físico e interacción reparatoria con sus adversarios después de un encuentro agresivo. Según De Waal, alrededor de un 40 por ciento de los enfrentamientos agresivos entre chimpancés terminan cuando, más o menos media hora más tarde, los involucrados establecen algún tipo de contacto conciliatorio (De Waal 1989a, 1989b).

En esos casos y en otros, los intentos sistemáticos y selectivos de reconciliación con los individuos con que se ha tenido un conflicto previo parecen requerir que los primates sean capaces de recordar qué tipo de interacciones agresivas han tenido en el pasado y quiénes fueron los involucrados (De Waal 1989b).

Los tres tipos de patrones conductuales examinados - las conductas selectivas hacia los parientes, los intercambios recíprocos y la reconciliación con los antagonistas - refuerzan una misma conclusión: entre las variadas competencias sociales de los primates no humanos se destaca la capacidad para identificar y reidentificar a individuos del grupo en distintos contextos, rastrearlos a lo largo del tiempo, registrar los cambios que les ocurren y modificar las expectativas y 
respuestas con respecto a ellos de un modo acorde. Entre las "sustancias" que los primates conceptualizan, ocupan un lugar privilegiado los semejantes con los cuales necesitan interactuar cotidianamente para garantizar la reproducción y la supervivencia.

\subsection{Identificación madre-hijo en leones marinos}

Pitcher y sus colegas (Pitcher et al. 2009) han estudiado detenidamente la competencia para el reconocimiento mutuo entre madres e hijos en leones marinos. Esta habilidad puede considerarse sumamente valiosa desde un punto de vista adaptativo, pues aumenta las posibilidades de supervivencia de los cachorros y evita que las madres derrochen esfuerzo y cuidados en crías ajenas. Por su parte, varios rasgos característicos del modo de vida de la especie vuelven aún más acuciante la necesidad de reidentificación exitosa entre madres e hijos. En primer lugar, estos mamíferos viven en colonias densamente pobladas, donde el riesgo de confundir a un individuo con otro es alto. A esto se suma que, poco después del nacimiento de los cachorros, las madres tienen que separarse de ellos por periodos breves (1 o 2 días) para buscar alimentos, y necesitan reunirse con sus crías al retornar. Finalmente, el riesgo para las crías es alto si no logran reunirse, pues las otras leonas marinas adultas tienden a ser agresivas con los infantes que no pertenecen a su familia.

En un experimento diseñado para medir el reconocimiento materno a través de las vocalizaciones, se hizo que algunos cachorros de leones marinos escucharan las vocalizaciones de sus madres junto con las de otras hembras. Los resultados mostraron que los cachorros respondían con mayor intensidad y rapidez a las vocalizaciones maternas que a las de las otras hembras desde los dos meses de edad (Pitcher et al. 2009). Lo que es más, en la mayoría de los casos, los cachorros ignoraron directamente los llamados de otras hembras. En contextos naturales, esta capacidad para el reconocimiento a distancia por medio de las vocalizaciones auditivas se complementa con la confirmación de la identidad de la madre y del cachorro gracias a la información sensorial que la vista, el tacto y el olfato brindan (Schusterman et al. 2002).

Otros rasgos llamativos del reconocimiento madre-hijo a través de las vocalizaciones en los leones marinos son su carácter aprendido y el que esa habilidad se conserve durante largo tiempo. Con respecto al primer punto, las madres aprenden a reconocer el llamado de sus cachorros a pocos días de su nacimiento. Éstos, en cambio, requieren un periodo más largo, que abarca entre los diez días y los dos 
meses posteriores al nacimiento. En cuanto al segundo punto, se ha estudiado que estos animales siguen reconociendo las vocalizaciones de madres e hijos al menos hasta dos años después del destete.

Este ejemplo muestra que también fuera del reino de los primates hallamos la capacidad de reconocer individuos de la propia especie. Cuando un león marino recién nacido reconoce las vocalizaciones de la madre a su regreso, se aproxima a ella y corrobora su identidad tocándola y oliéndola; está identificando a mamá otra $v e z$, por vías sensoriales diferentes, como resultado de un proceso gradual de aprendizaje. Aunque su concepción sobre la madre -i.e., las representaciones que se forma al respecto, la información que almacena, las inferencias que puede realizar y los medios que emplea para reconocerla - sea muy diferente de la de un humano, también los leones marinos cuentan, de acuerdo con la teoría de Millikan, con un concepto básico de mamá. ${ }^{14}$

\subsection{Reconocimiento social en hienas}

Las hienas moteadas forman parte de sociedades semejantes a las de los primates, tanto por su tamaño y estructura como por sus patrones de competencia y cooperación. Estos animales pertenecen a grupos sociales permanentes, llamados clanes, compuestos por entre seis y noventa miembros. Los miembros de un mismo clan establecen relaciones duraderas entre sí, defienden de modo cooperativo el territorio que comparten y crían juntos a sus cachorros en una guarida común.

Además, como los primates, las hienas muestran distintos comportamientos indicadores de la capacidad para reconocer miembros individuales de su grupo o clan. He aquí dos de los más llamativos:

i) Como sucede con los monos vervet (Cheney y Seyfarth 1990), las hienas distinguen las vocalizaciones de sus crías y de las crías ajenas y responden de manera diferenciada a las primeras, lo cual sugiere que cuentan con la capacidad para individualizar a sus hijos por este medio (Holekamp y Engh 2002, Holekamp

\footnotetext{
${ }^{14}$ Este caso, como otros de reidentificación de individuos en distintas especies, supone la integración de información recogida a lo largo del tiempo a través de distintas modalidades sensoriales. Por ello, a pesar de su interés, propuestas como la de Pylyshyn (2009), que postula capacidades no conceptuales circunscritas al ámbito de la percepción visual para el rastreo de objetos particulares, no bastan para explicar la evidencia aquí presentada. A esto se suma que, en muchos casos, la información proveniente de distintas modalidades se preserva a lo largo del tiempo. Esto conlleva cierta distancia respecto de la estimulación inmediata, lo que nos da razones para afirmar que estamos ante habilidades conceptuales y no meramente perceptuales.
} 
et al. 2007). Datos experimentales adicionales indican que las hienas poseen una capacidad análoga para reconocer a otros individuos de su grupo por medio de vocalizaciones.

ii) Al igual que muchos primates, después de una pelea o un conflicto, las hienas suelen ejecutar (entre 10 y 15 por ciento de las veces) conductas reconciliatorias, destinadas a reparar el vínculo social dañado y/o a reducir la tensión grupal. Usualmente, la reconciliación consiste en que el perdedor levanta una de sus patas en un "saludo" amistoso dirigido a su antiguo oponente (Holekamp y Engh 2002, Holekamp et al. 2007). Por otra parte, es mayor la frecuencia de conductas reconciliatorias entre individuos sin parentesco, algo que puede deberse, según estos autores, a que esperan encontrar una mayor tolerancia a las agresiones entre los parientes. Todo esto parece indicar que las hienas pueden identificar y recordar con qué individuos han tenido intercambios agresivos, e integrar esa información con otra relativa a si mantienen con ellos un vínculo de parentesco.

La evidencia seleccionada permite pensar que, como muchos primates no humanos, las hienas son capaces de identificar a otros individuos de su clan, recordar la interacción cooperativa previa que han tenido con ellos y ajustar su comportamiento de modo acorde. Henos aquí, pues, con razones empíricas para concluir que también estos animales cuentan con algunos conceptos de sustancias.

Aunque breve y fragmentaria, la revisión empírica de esta sección avala la idea de que los conceptos de sustancia no son patrimonio exclusivo de los animales humanos, sino que forman parte de las herramientas cognitivas de distintas especies. Ahora bien, ¿son éstos los únicos conceptos que podemos atribuir a los animales sin lenguaje?, ¿o podemos también, en ocasiones, atribuir a algunas especies la habilidad más compleja para identificar propiedades?

\section{Animales que identifican propiedades}

A diferencia de lo que ocurre con los conceptos de sustancia, resulta más difícil atribuir conceptos de propiedades a animales sin lenguaje pues, como ya señalé, su posesión involucra modificaciones importantes de nuestras habilidades cognitivas, entre las que destacan un aumento de las potencialidades inferenciales y combinatorias, la capacidad para pensar en objetos novedosos y la posibilidad de diferenciar la propiedad que se identifica de otras contrarias, que no pueden presentarse simultáneamente en una misma sustancia. Así, 
para defender que algunos animales identifican propiedades es necesario hallar evidencia conductual que refleje la posesión de esas capacidades cognitivas adicionales. Tal tarea excede el marco de este trabajo y, por lo tanto, sólo examinaré aquí algunas investigaciones que tornan sugerente la hipótesis de que dos especies animales - los chimpancés y los cuervos de Nueva Caledonia - poseen habilidades para identificar algunas propiedades funcionales.

\subsection{Identificación de propiedades funcionales en chimpancés}

La etología cognitiva brinda evidencia cuidadosa acerca de que diversos primates emplean numerosas herramientas en entornos naturales. Los chimpancés, además, construyen algunas de estas herramientas; esto es, crean objetos previamente inexistentes y necesarios para alcanzar un fin. Un ejemplo conocido de construcción de herramientas es la manufactura de "cañas de pescar" con palitos, las cuales emplean para capturar hormigas y termitas, o para extraer restos de nuez escondidos en la cáscara de ese fruto.

Las observaciones etológicas muestran que los chimpancés modifican esas herramientas en función de la tarea específica en la cual las van a emplear. Por ejemplo, los huecos de una colmena o un hormiguero son más amplios y profundos que los intersticios de una cáscara de nuez. De un modo que se ajusta a estas diferencias en amplitud y profundidad, los chimpancés de Tai construyen sus "cañas de pescar" cortando palitos de dos tamaños distintos: unos más cortos y delgados para extraer la carne de la nuez, y otros más largos y gruesos para "pescar" hormigas y extraer miel. Esto puede interpretarse como un primer indicador de que los chimpancés distinguen, de algún modo, esos pares de propiedades contrapuestas y son capaces de determinar cuál de ellos necesitan según la tarea que van a realizar.

Dependiendo del empleo futuro, la ramita puede sufrir una o más de las siguientes modificaciones: a) se arranca del árbol empleando manos y dientes; b) se corta de un largo específico; c) se le quitan las hojas o la corteza con dientes y manos, o d) se aguza la punta con los dientes. Puesto que se emplean para meterlas en huecos, las ramitas tienen que reunir ciertos rasgos: ser rectas y del largo y el ancho apropiados. Es importante añadir a esto que, en casi todos los casos, los chimpancés preparan de antemano la herramienta que van a utilizar, la alteran hasta que presenta las características adecuadas para la tarea y aplican muy pocas modificaciones después de su primer uso. Todo ello sugiere que estos animales poseen "una 
idea precisa de la forma que el objeto debe tener para ser una herramienta" (Boesch y Boesch 1990, p. 95).

El que los chimpancés modifiquen previamente cierto material "en bruto" hasta crear una herramienta adecuada para la tarea específica que van a realizar indica que son capaces de identificar de algún modo las propiedades funcionales que debe tener el artefacto en cuestión. Más aún, sugiere que poseen alguna capacidad para pensar en tales propiedades con independencia de cualquier sustancia específica, pues no se limitan a detectar su presencia en un objeto ya existente, sino que modifican activa y flexiblemente ciertos materiales para conferirles propiedades que no poseen, pero que resultan necesarias para sus propósitos.

Sería conveniente, sin duda, contar con más evidencia con respecto a la capacidad de los chimpancés para detectar esas propiedades en distintas sustancias. Con ese fin, podría ser interesante realizar estudios para establecer si, cuando se les presentan materiales diferentes, pero con las propiedades funcionales adecuadas (por ejemplo, una barra de plástico o un alambre), son capaces de detectar tales rasgos y emplear los nuevos materiales para la pesca de hormigas o la búsqueda de miel.

4.2. Identificación de propiedades funcionales en pájaros: el caso de los cuervos de Nueva Caledonia

Los cuervos de Nueva Caledonia parecen ser los únicos animales fuera de la esfera de los mamíferos que construyen y emplean herramientas en contextos naturales y en cautiverio. La pregunta que nos interesa aquí es si estas aves son capaces de identificar las propiedades que hacen de ciertos objetos herramientas útiles para alcanzar sus objetivos. Para evaluar este punto, resultan de especial interés las investigaciones llevadas a cabo por Kalcenik y colegas (Weir y Kalcenik 2006, Bluff et al. 2007) con dos cuervos: Betty y Abel.

En un primer experimento, los investigadores pusieron al alcance de los cuervos herramientas de diez largos diferentes y colocaron alimentos ubicados a distintas profundidades dentro de un tubo transparente en posición horizontal. Los sujetos seleccionaron las herramientas del largo apropiado para retirar cada alimento desde la primera prueba, y sólo se equivocaron en un 13 por ciento de los casos.

En un segundo experimento, los cuervos debían seleccionar herramientas del ancho apropiado. En este caso, frente a los sujetos se colocaron recipientes con agujeros de distintos tamaños, por los que debían introducir la herramienta del ancho adecuado para poder ob- 
tener la comida. En una primera prueba, se le ofrecieron a Betty tres herramientas de distinto grosor, ante las cuales mostró una marcada tendencia a elegir la más delgada (que servía para retirar la comida en todos los casos). En estudios en los que los cuervos debían construir una herramienta para insertarla en huecos más o menos anchos se obtuvieron resultados similares. En todos los casos, esas aves mostraron una clara tendencia a construir herramientas del tamaño apropiado para cada ocasión. Más aún, en 27 de las 30 pruebas realizadas lograron dar a la herramienta el grosor adecuado antes de emplearla por primera vez.

Estos estudios indican que los cuervos registran selectivamente las dimensiones de las herramientas que para ellos resultan relevantes desde un punto de vista funcional, como largo y ancho. Más aún, como los chimpancés, los cuervos se muestran capaces de construir herramientas del ancho adecuado para un propósito (retirar comida) antes de ponerlas a prueba, lo cual sugiere que se representan la propiedad requerida, aun cuando ésta no se instancie todavía en la sustancia (el material en bruto) con la que interactúan.

Aunque las anteriores son buenas razones para sospechar que los cuervos de Nueva Caledonia identifican propiedades, un tercer grupo de estudios aporta evidencia aún más contundente al respecto. En ellos, un cuervo hembra (la ya mencionada Betty) logró, de modo espontáneo y sistemático, curvar un trozo de alambre hasta darle la forma de un gancho y emplear esa herramienta para extraer un pequeño balde con comida que se encontraba dentro de un tubo en posición vertical. Las acciones de Betty al momento de construir esta herramienta llamaron la atención de los investigadores por parecer:

i) deliberadas: Betty llevó a cabo acciones bien delimitadas, que no eran resultado de una manipulación general y azarosa del material, y siempre empleó el gancho para obtener el alimento inmediatamente después de fabricar la herramienta.

ii) específicas: Betty nunca realizó una acción similar en otros contextos y, aunque usó técnicas diversas, las herramientas finales siempre se caracterizaron por su forma curva.

iii) novedosas: las acciones realizadas para curvar el alambre no se asemejaban a ninguna de las observadas en estas aves en contextos naturales (Weir y Kalcenik 2006).

Tiempo después se realizó otra prueba, en la cual Betty tenía que construir una herramienta curva para poder extraer el balde del tubo 
plástico, pero con un material diferente: tiras de aluminio en lugar de alambre. Para poder curvar ese material, Betty tuvo que inventar una nueva técnica: sostenía la tira por el extremo que había que doblar y no por el lado opuesto, como hacía en el caso del alambre. El ave logró llevar a cabo estas modificaciones con éxito y llamativa rapidez (desde la tercera prueba).

Estos estudios brindan evidencia adicional de que los cuervos de Nueva Caledonia son capaces de identificar qué propiedad necesitan de una herramienta, representarla mentalmente aun cuando no esté presente en el entorno y buscar su instanciación (en estos casos, construyéndola ellos mismos) en las más diversas sustancias.

\section{Conclusiones}

La variante de pragmatismo conceptual que defiendo a lo largo de este trabajo no es, sin duda, la única alternativa teórica atendible para abordar el problema de la posesión de conceptos en los animales no humanos, pero sí parece una estrategia alternativa especialmente interesante pues, según vimos, este enfoque considera que las habilidades conceptuales son múltiples y heterogéneas. De allí que, sin pretender exhaustividad, haya diferenciado aquí dos tipos de habilidades conceptuales — la identificación de sustancias y la identificación de propiedades - que desempeñan funciones cognitivas diferentes y entrañan diversos niveles de complejidad. Tal distinción posibilita un análisis detallado de la evidencia empírica, que no se limita a atribuir conceptos sin más a distintas especies, sino que atiende a los tipos de conceptos atribuidos en cada caso y a las diferencias conductuales y cognitivas que cada uno de ellos conlleva.

Entre las virtudes de esta propuesta se cuenta, por lo tanto, el permitir una extensión gradual y matizada de las habilidades para identificar sustancias y propiedades a los animales no humanos. Según vimos, en la medida en que estas habilidades satisfacen - también en grados diversos - los requisitos para calificarlas de genuinamente conceptuales, podemos concluir que al menos algunas especies no humanas poseen algunos conceptos. ${ }^{15}$

\footnotetext{
${ }^{15}$ Quiero agradecer a todos los integrantes del grupo Conceptos y Percepción, en especial a Mariela Aguilera y Daniel Kalpokas, por sus cuidadosas lecturas y comentarios a versiones previas de este trabajo.
} 


\section{BIBLIOGRAFÍA}

Allen, C., 1999, “Animal Concepts Revisited”, Erkenntnis, vol. 51, no. 1, pp. 33-40.

Bekoff, M., C. Allen y G.M. Burghardt (comps.), 2002, The Cognitive Animal: Empirical and Theoretical Perspectives on Animal Cognition, The MIT Press, Cambridge, Mass.

Bermúdez, J.L., 2003, Thinking without Words, Oxford University Press, Oxford.

Bluff, L.A, A.A.S. Weir, C. Rutz, J.H. Wimpenny y A. Kacelnik, 2007, "Tool-Related Cognition in New Caledonian Crows", Comparative Cognition and Behavior Reviews, vol. 2, pp. 1-25.

Boesch, C. y H. Boesch, 1990, "Tool Use and Tool Making in Wild Chimpanzees", Folia Primatologica, vol. 54, nos 1-2, pp. 86-99.

Call, J., 2006a, "Descartes' Two Errors: Reason and Reflection in the Great Apes", en Hurley y Nudds 2006, pp. 219-234.

—, $2006 \mathrm{~b}$, "Inference by Exclusion in the Great Apes: The Effect of Age and Species", Animal Cognition, vol. 9, no. 4, pp. 393-403.

Camp, E., 2009, "Putting Thoughts to Work: Concepts, Stimulus-Independence and the Generality Constraint", Philosophy and Phenomenological Research, vol. 78, no. 2, pp. 275-311.

Cangelosi A. y D. Parisi, 1998, "Concepts in Artificial Organisms. A Commentary on Millikan", Behavioral and Brain Sciences, vol. 21, no. 1, pp. 68-69.

Carruthers, P., 2009, "Invertebrate Concepts Confront the Generality Constraint (and Win)", en R. Lurz (comp.), The Philosophy of Animals Minds, Cambridge University Press, Cambridge, pp. 89-107.

Cheney D.L. y R.M. Seyfarth, 2007, Baboon Metaphysics: The Evolution of a Social Mind, The University of Chicago Press, Chicago.

- 1990, How Monkeys See the World. Inside the Mind of Another Species, The University of Chicago Press, Chicago.

Child, W., 1994, Causality, Interpretation and the Mind, Clarendon Press, Oxford.

Cords, M., 1997, "Friendships, Alliances, Reciprocity and Repair", en A. Whiten y R.W. Byrne (comps.), Machiavellian Intelligence II: Extensions and Evaluations, Cambridge University Press, Cambridge, pp. 24-49.

Danón, L., 2011, "Normatividad doxástica en animales no humanos", Analítica, vol. 5, pp. 9-29.

— 2008, "Discriminar, identificar, conceptualizar", en G. Agüero, D. Vera y L. Urtubey (comps.), Conceptos, creencia y racionalidad, Brujas, Córdoba, pp. 187-194.

Davidson, D., 1982, “Animales racionales", en Subjetivo, intersubjetivo, objetivo, trad. O. Fernández Prat, Cátedra, Madrid, pp. 141-155. 
De Waal, F., 1989a, Chimpanzee Politics: Power and Sex among the Apes, Johns Hopkins University Press, Baltimore.

— $1989 \mathrm{~b}$, Peacemaking among Primates, Harvard University Press, Cambridge, Mass.

Duhau, L., 2009, "Conceptuality and Generality: A Criticism of an Argument for Concept Dualism", Crítica. Revista Hispanoamericana de Filosofía, vol. 41, no. 123, pp. 39-63.

Evans, G., 1982, The Varieties of Reference, Clarendon Press, Oxford.

Fodor, J., 1995, "Concepts: a Potboiler", Philosophical Issues, vol. 6, pp. 124.

Glock, H.J., 2008, La mente de los animales. Problemas conceptuales, trad. V.M. Santamaría Navarro, KRK Ediciones, Oviedo.

Holekamp, K.E. y A.L. Engh, 2002, "Field Studies of Social Cognition in Spotted Hyenas", en Bekoff, Allen y Burghardt 2002, pp. 371-378.

Holekamp, K.E., S.T. Sakai y B.L. Lundrigan, 2007, "Social Intelligence in the Spotted Hyena (Crocuta crocuta)", Philosophical Transactions of the Royal Society B, vol. 362, no. 1480, pp. 523-538.

Hurley, S., 2006, "Making Sense of Animals", en Hurley y Nudds 2006, pp. 139-171.

Hurley S. y M. Nudds (comps.), 2006, Rational Animals?, Oxford University Press, Oxford.

Kapsalis, E., 2004, "Matrilineal Kinship and Primate Behavior", en B. Chapais y C.M. Berman (comps.), Kinship and Behavior in Primates, Oxford University Press, Oxford, pp. 153-176.

Lovibond, S., 2006, "Practical Reason and Its Animal Precursors", European Journal of Philosophy, vol. 14, no. 2, pp. 262-273.

McDowell, J., 1994, Mente y mundo, trad. M.Á. Quintana Paz, Sígueme, Salamanca.

Millikan, R., 2000, On Clear and Confused Ideas, Cambridge University Press, Cambridge.

-, 1998a, "A Common Structure for Concepts of Individuals, Stuffs, and Real Kinds: More Mama, More Milk, More Mouse", Behavioral and Brain Sciences, vol. 21, no. 1, pp. 55-65.

, 1998b, "With Enemies Like These I Don't Need Friends", Behavioral and Brain Sciences, vol. 21, no. 1, pp. 89-100.

- 1984 , Language, Thought and Other Biological Categories, The MIT Press, Cambridge, Mass.

Pitcher, B.J., H. Ahonen, R.G. Harcourt e I. Charrier, 2009, “Delayed Onset of Vocal Recognition in Australian Sea Lions Pups (Neophoca cinerea)", Naturwissenschaften, vol. 96, no. 8, pp. 901-909.

Price, H., 2010, "One Cheer for Representationalism", en R.E. Auxier y L.E. Hahn (comps.), The Philosophy of Richard Rorty, Open Court, Chicago, pp. 269-289. 
Pylyshyn, Z.W., 2009, "Perception, Representation and the World: the FINST that Binds", en D. Dedrick y L.M. Trick (comps.), Computation, Cognition and Pylyshyn, The MIT Press, Cambridge, Mass., pp. 3-48.

Rorty, R., 1997, ¿Esperanza o conocimiento? Una introducción al pragmatismo, trad. E. Rabossi, Fondo de Cultura Económica, Buenos Aires.

- - 1991, Objectivity, Relativism, and Truth: Philosophical Papers, Cambridge University Press, Cambridge.

Ryle, G., 1949 (1967), El concepto de lo mental, trad. E. Rabossi, Paidós, Buenos Aires, 1967.

Schusterman, R.J., C.R. Kastack y D. Kastak, 2002, "The Cognitive Sea Lion: Meaning and Memory in the Lab and in Nature", en Bekoff, Allen y Burghardt 2002, pp. 217-228.

Shea, N., 2005, On Millikan, Thomson Wadsworth, Belmont.

Ventura, R., B. Majolo, N.F. Koyama, S. Hardie y G. Schino, 2006, "Reciprocation and Interchange in Wild Japanese Macaques: Grooming, Co-Feeding and Agonistic Support", American Journal of Primatology, vol. 68, no. 12, pp. 1138-1149.

Vigo, R. y C. Allen, 2009, "How to Reason without Words: Inference as Categorization", Cognitive Processing, vol. 10, no. 1, pp. 51-88.

Watanabe, S. y L. Huber, 2006, “Animal Logics: Decisions in the Absence of Human Language", Animal Cognition, vol. 9, no. 4, pp. 235-245.

Weir, A.A.S. y A. Kacelnik, 2006, "A New Caledonian Crow (Corvus moneduloides) Creatively Re-Designs Tools by Bending or Unbending Aluminum Strips", Animal Cognition, vol. 9, no. 4, pp. 317-334.

Recibido el 26 de julio de 2011; revisado el 23 de septiembre de 2012; aceptado el 4 de diciembre de 2012. 